\title{
A heuristic approach to feasibility verification for truck loading
}

\author{
Vinicius Gandra \\ KU Leuven, Department of Computer Science, CODeS \\ Belgium \\ vinicius.gandramartinssantos@kuleuven.be \\ Tony Wauters \\ KU Leuven, Department of Computer Science, CODeS \\ Belgium
}

\begin{abstract}
This paper introduces an industrial decision problem emerging from daily operations of a company which provides its clients in Europe and Latin America with machine rentals. The company faces several challenges in distribution of these machines using a fleet of trucks. The trucks with a non-convex loading surface are loaded with multiple machines of irregular shapes to be picked up or delivered to/from customer or depot locations. Within the algorithmic framework utilized by the company, for each route generated, one must verify whether or not it is possible to load the corresponding machines onto the truck without violating certain restrictions.

The aim of this paper is to develop a competitive and automated algorithm capable of efficiently classifying load plans in a very restrictive scenario. For this purpose, we extend a well-known heuristic algorithm from the two-dimensional (2D) packing literature and compare its performance with two other methods: the original 2D packing method and the current method employed by the company in practice. The computational experiments are performed in a set of candidate load plans provided by the company. We observe that the newly introduced adaptation outperforms the other methods and correctly classifies $90 \%$ of the given load plans provided.
\end{abstract}

\section{CCS CONCEPTS}

- Applied computing $\rightarrow$ Supply chain management; Transportation; • Computing methodologies $\rightarrow$ Cross-validation; Classification and regression trees.

\section{KEYWORDS}

Automation, decision problems, two-dimensional packing, heuristics

\section{ACM Reference Format:}

Vinicius Gandra, Hatice Çalık, Tony Wauters, and Greet Vanden Berghe. 2021. A heuristic approach to feasibility verification for truck loading. In 2021 Genetic and Evolutionary Computation Conference Companion (GECCO

Permission to make digital or hard copies of all or part of this work for personal or classroom use is granted without fee provided that copies are not made or distributed for profit or commercial advantage and that copies bear this notice and the full citation on the first page. Copyrights for components of this work owned by others than ACM must be honored. Abstracting with credit is permitted. To copy otherwise, or republish, to post on servers or to redistribute to lists, requires prior specific permission and/or a fee. Request permissions from permissions@acm.org.

GECCO '21 Companion, July 10-14, 2021, Lille, France

(c) 2021 Association for Computing Machinery.

ACM ISBN 978-1-4503-8351-6/21/07 . \$ \$15.00

https://doi.org/10.1145/3449726.3463184

\author{
Hatice Çalık \\ KU Leuven, Department of Computer Science, CODeS \\ Belgium \\ hatice.calik@kuleuven.be \\ Greet Vanden Berghe \\ KU Leuven, Department of Computer Science, CODeS \\ Belgium
}

'21 Companion), fuly 10-14, 2021, Lille, France. ACM, New York, NY, USA, 6 pages. https://doi.org/10.1145/3449726.3463184

\section{INTRODUCTION}

The feasibility verification and machine packing problem (FVMP) introduced in this paper emerges from a necessity to automate decisions concerning whether or not a given set of machines can be loaded onto a non-convex truck loading surface before physically performing those loading operations in reality. To better appreciate how and why this problem is relevant, we will first provide a broader description of the operations of the company in question.

The company which inspired this research owns a set of nonidentical machine types which they rent to their customers. A fleet of identical trucks is employed by the company to pickup and deliver machines from/to the depots or customer locations on a daily basis. Each customer uses the requested machines for a number of days before requiring those machines to be picked up once the rental period ends. On a given day, it is possible to have both pickup and delivery requests from different customers. A machine to be delivered to a customer does not necessarily depart from a depot, but can instead be picked up from another customer on the same day. In order to minimize transportation costs, it is desirable to transport multiple machines in the same truck whenever possible and profitable. However, this requires ensuring the feasibility of loading multiple machines in a truck before it departs on its journey and, ideally, during the planning phase of the routes.

Most of the considered machines have irregular shapes with arms or forks, as illustrated in Figure 1. The total length of these

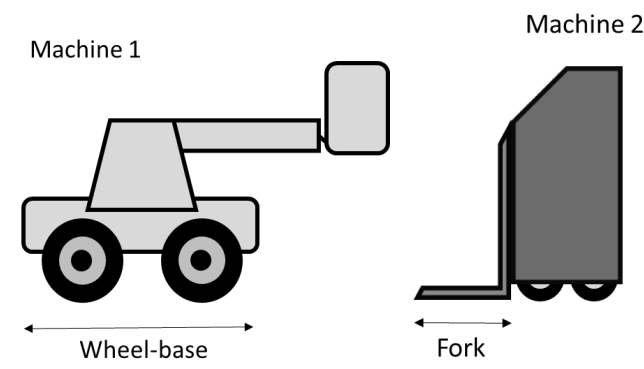

Figure 1: Machines with an arm (machine 1) and with a fork (machine 2).

machines is divided into its wheelbase and these special parts. These 
special parts allow the vertical projection of machines to overlap with others when loading them onto trucks. Other machines have their length completely defined by their wheelbase, as illustrated in Figure 2. During transportation, the machines may assume different forms by (un)folding these arms or raising their forks to better position themselves on the truck's loading surface.
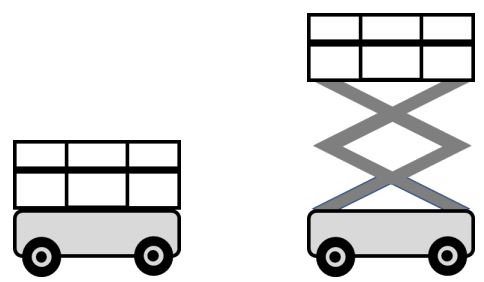

Figure 2: Machines with a lift which can be folded and unfolded.

Trucks have a regular surface on which any machine can be loaded. An additional loading surface can be occupied over the gooseneck by only a subset of machines and by respecting the relevant restriction which will be detailed in Section 2.

The company which introduced this problem employs a routing algorithm for scheduling the pickup and delivery of machines. To validate the loading feasibility at each pickup location, the routing algorithm considers only a scalar value to represent the area occupied by each machine on the truck loading surfaces. These values, referred to as the truck occupation percentages, are calculated based on the observations and knowledge of truck drivers. It is thus far from being an automated process at present. Henceforward we address to this method as the predicted percentage method (PPM). Although the PPM percentages are calculated by taking into account the dimensions of the machines, these scalar values are not sufficiently accurate to separate feasible and infeasible loading combinations. As a result, the routing algorithm with the PPM ends up scheduling many routes which are infeasible with respect to loading constraints which results in delayed deliveries and the use of additional trucks.

The company in question owns nearly 1700 different types of machine and new machine types are constantly being added to their inventory. Therefore, it is impractical to physically enumerate all possible feasible loading configurations (with all subsets of machines) in a pre-processing step prior to the routing algorithm. In fact, since routing algorithms often perform hundreds of thousands iterations, see for instance the genetic algorithm by Vidal et al. [13], any routing feasibility verification should be carried out as fast as possible to avoid excessive runtimes. The challenge is thus to develop a more effective yet fast way of checking the feasibility of truck load plans.

The algorithm introduced in Section 4 considers machines as twodimensional (width, length) items and a truck with two different loading surfaces. The foundation for the heuristic algorithm to solve this very challenging problem is the two-dimensional best-fit heuristic of Burke et al. [2]. However, given the problem's special restrictions, the best-fit heuristic alone is not enough to serve as an accurate feasibility check. In this paper we propose an extension of the best-fit heuristic which is able to handle tailored constraints to better adapt to the company's scenario.

In order to maintain the developed heuristic's efficiency, we make a series of assumptions and simplifications regarding the real-world problem. Although rarely, due to these assumptions, the algorithm can yield load plans which are infeasible in reality. Therefore, as a means of evaluating the effectiveness of the algorithm, we conduct a classification study. The motivation for this classification study is briefly outlined in what follows.

The company provided us with real-world data concerning their machines and potential load plans. Prior to executing any packing method, we do not have any knowledge on the feasibility of a potential load plan in reality. Therefore, there are two possible outcomes in reality: (i) a positive outcome, that is, there exists a feasible loading scheme and (ii) a negative outcome, that is, there exists no feasible loading scheme. In theory, an exact method for solving the problem with all its given elements and without any simplifications or assumptions always returns the actual reality. In that case, the algorithmic outcomes can be classified into two types: true positive (the method finds a feasible loading scheme and a feasible scheme exits in reality) or true negative (the method terminates with no feasible schemes and there exists no feasible scheme in reality). The outcomes of a heuristic algorithm for the same problem include an additional classification type: false negative (the method terminates with no feasible schemes while there exits a feasible scheme in reality).

Finally, a heuristic method solving a simplified version of the problem with certain assumptions can provide a fourth type of classification: false positive (the method terminates with a feasible loading scheme while there exists no feasible scheme in reality). The algorithm we introduce in this paper and the two other algorithms used for computational comparison all fall into this last group of heuristic methods. In these experiments, our approach significantly outperforms the PPM correctly classifying $90 \%$ of the potential load plans (the percentage of true positives and true negatives), which is considered highly satisfactory by the company.

The remainder of this paper is organized as follows. Section 2 describes the problem in detail. Section 3 briefly reviews the most relevant studies in the literature. Section 4 details the algorithm we develop, while Section 5 presents the results of the computational study we conducted. Finally, Section 6 concludes the paper and outlines some future research directions.

\section{PROBLEM DESCRIPTION}

The feasibility verification and machine packing problem (FVMP) consists of two main components: a truck and a set of machines. Trucks have two areas onto which machines can be loaded. The first is simply the regular loading area, which is 10 meters long and 2.5 meters wide. The second loading area, which is referred to as the gooseneck, extends the length of the truck by an additional 3 meters. As illustrated in Figure 3, the gooseneck is not at the same height as the regular loading surface and therefore not every machine can occupy this part of the truck.

Certain machines can occupy the gooseneck while positioned in the regular loading surface, as illustrated in Figure 4. Other machines can be entirely placed on the gooseneck if their length 


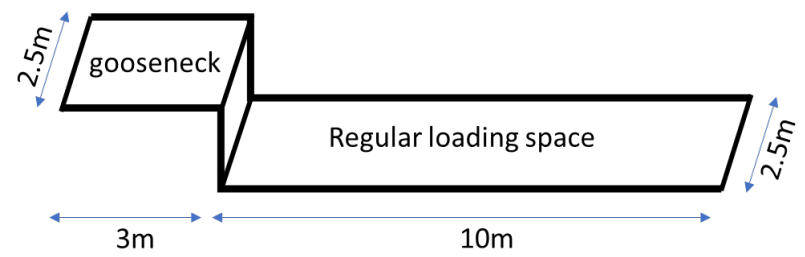

Figure 3: The gooseneck and the regular loading surface of the truck.

is not greater than that of the gooseneck and if they have wheels enabling them to climb onto the gooseneck with the assistance of a portable or build-in ramp (see Figure 5).

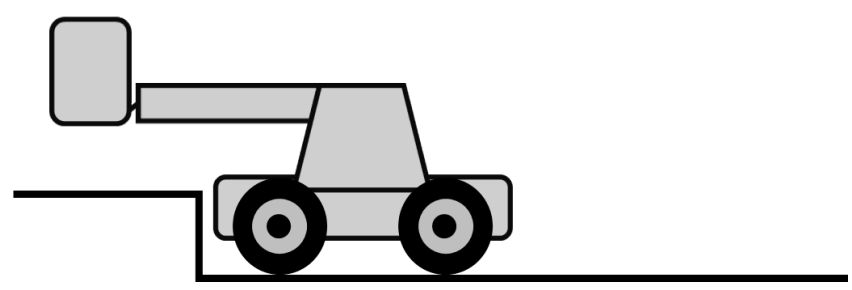

Figure 4: Illustration of a machine partially occupying the gooseneck.
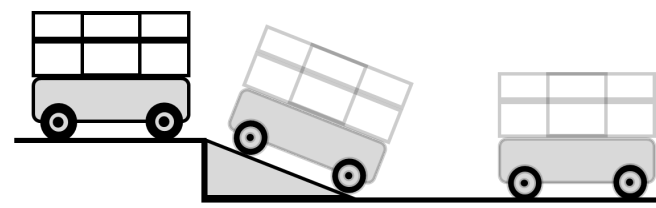

Figure 5: Illustration of a machine placed entirely on the gooseneck.

Most machines do not have a fixed shape. In fact, many machines have articulated arms which can be folded into several forms, some of which can be cuboid. Meanwhile, some machines have forks which can partially go under the arms or wheel base of other machines (see Figure 6). Two length values are considered per machine: the length of the wheelbase (WBL) and the total length (TL). Therefore, a partial overlapping of the projection of the machines is sometimes possible.

Figure 7 illustrates just one example of such overlap. In this example machine 1 has a small wheelbase when compared to its total length. However, not all of its extra length can be overlapping another machine. While part of the arm is suspended above the loading surface allowing overlap with shorter machines, the cage (on the end of its articulated arm) is located at ground level forbidding any overlap. Thus, only a portion of the difference between the machine's wheelbase length and total length may overlap with another machine. In Figure 7, by placing machine 2 over the gooseneck a feasible solution is possible without overlapping. On the other hand, the gooseneck in Figure 8 is occupied and only by overlapping machines a feasible load plan would be possible.

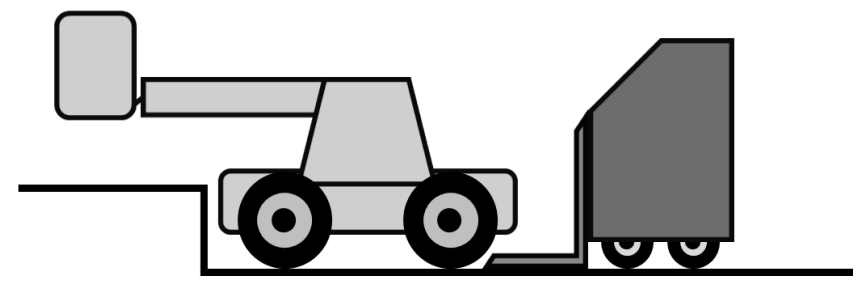

Figure 6: Illustration of two machines partially overlapping, where the fork of one machine goes under the wheel base of the other.

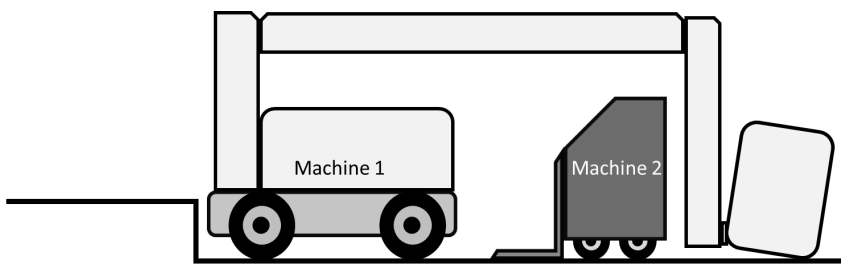

Figure 7: Illustration of an overlap where one machine is placed entirely under the arm of another.

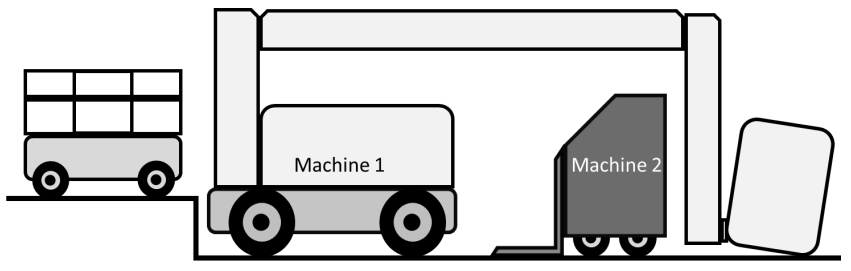

Figure 8: A loading scheme with overlap and use of the gooseneck.

A feasible load plan for the FVMP must take into account the following constraints:

- All machines must fit onto the loading surface of the truck.

- For safety reasons there must be open space between two machines that are positioned side by side.

- All load plans are subjected to maximum weight limits.

- The wheelbase of machines should never overlap.

- Machines must not be stacked.

- Given the need to drive the machines onto the truck, a fixed orientation is defined. Machines either drive forward onto the truck or reverse onto the truck, but never sideways.

\section{RELATED WORK}

The FVMP combines several components from different Cutting and Packing problems [14]. However, it is difficult to fit the FVMP into a single problem category without over-generalizing. In relation to the typology provided by Wäscher et al. [14], the FVMP is a three-dimensional (3D) packing problem with a single large object (all dimensions fixed) and a few small heterogeneous items of irregular shapes. The FVMP is a feasibility problem where the goal is to decide whether or not a feasible packing of the small 
items into the larger object is possible. Therefore, the FVMP does not aim to minimize or maximize any objective function.

The FVMP can be considered as a variant of the container loading problem (CLP) where rectangular-shaped items, which are often referred to as boxes, are placed in a cuboid container [1]. In the 2D case of the CLP, items can neither be stacked on top of each other nor are they allowed to overlap. A typical example of the 2D-CLP is the pallet loading problem [12]. Stacking is allowed in the 3D version of the problem [15].

The irregular shape of machines in the FVMP resembles the 3D irregular packing problem where items of irregular shapes are packed into a cuboid [9]. The machines can also be treated as tetris-like items [4]. However, the FVMP differs from all the aforementioned problems as its loading surface is not convex. The machines cannot be suspended in the air and should be standing on their wheels with a fixed orientation. Additionally, the structure of the truck's loading surface introduces additional restrictions which do not exist in the aforementioned studies. Although this structure resembles a multi-compartment CLP variant [6], the fact that some machines can partially occupy both loading surfaces distinguishes the FVMP from the traditional multi-compartment CLP problems.

Despite the distinguishing properties mentioned earlier, it is clearly possible to utilize or adapt some of the methods from the irregular 3D packing literature to address the FVMP. However, we foresee that these methods would require much more processing time which is undesirable as it makes the overall routing and feasibility checking process highly inefficient.

The company is using an off-the-shelf vehicle routing algorithm for the pickup and delivery of machines while the FVMP is solved for each route to ensure its feasibility or to detect infeasibilities and re-route accordingly. However, one might consider tackling these two problems, namely the routing and the loading problems, with the help of integrated methods. When we examine the literature, we see examples of such methods for combined vehicle routing and container loading problems with 2D or 3D rectangular-shaped items. To the best of our knowledge, no such method is readily available for solving the combined irregular 3D loading and the pickup and delivery problem.

For interested readers, we refer to Leao et al. [7] for a recent review on the irregular packing problems including those with irregular containers, to do Nascimento et al. [3] for a review of the CLP with practical constraints and to Pollaris et al. [11] for a review of combined CLP and vehicle routing problems.

\section{LOAD PLAN METHOD}

This paper introduces the load plan method (LPM) which uses a placement heuristic and determines the placement (position) of items inside trucks. This method should be capable of being efficiently embedded within the company's routing algorithm and will be called frequently when creating routes. Therefore, it is important for the LPM to produce high quality load plans in low computational runtimes.

An adaptation of the best-fit placement heuristic proposed by Burke et al. [2] is used in this paper which follows the efficient implementation by Imahori and Yagiura [5]. This approach was chosen given its simplicity to implement, computational performance and the high-quality solutions generated. The best-fit heuristic is a constructive method. Given a list of items and a container, the method finds the position furthest from the rear of the container and places the item that fits best in it. Items are iterated over in a predetermined order until one item can be successfully placed. Once an item is inserted, the furthest position is updated and the placement continues until all items have been loaded.

The implementation in this paper sorts machines based on their width, length and area. Ties between machines with the same dimension are broken by either length or width. When placing a machine inside the truck, one of two insertion policies can be followed: insert the machine next to the longest neighbor machine or insert the machine next to the neighbor with the most similar length. The LPM combines each machine ordering with an insertion policy and returns the first feasible load plan generated.

To further adapt our load plan method so that it can accommodate the specific characteristics of the problem presented in Section 2, we introduce the following parameters:

- Extra width (exW): for safety reasons, a certain amount of extra space must be preserved between machines when loaded side by side. In order to ensure this safety distance is respected, we extend machines' width by exW if their original width is below a threshold $\max _{w}$. This extension is not needed for wider machines as it will never be possible to load multiple wide machines side by side.

- Minimum machine length required to partially occupy the gooseneck (min_gNeck): given the difference in height, only large machines are able to occupy both the loading surface and the gooseneck. Parameter min_gNeck indicates how big a machine must be to be positioned partially on the gooseneck and partially on the loading surface.

- Maximum weight (max_weight): the considered machines are often large and heavy, which may lead to infeasible load plans due to their total weight instead of the physical space they occupy. We set max_weight based on the maximum weight regulations in effect in the country where the company provides services.

- Reduced length percentage (reduced_len\%): in addition to the total length, the wheelbase length is provided. While the wheelbase indicates a portion of the machine which can never overlap with another, the machine's arms or forks can often overlap depending on the combination of machines. Using reduced_len\% we define a new machine length to be considered which is outlined in Equation (1). This new length is equal to the total length if reduced_len $\%=0$ and equals the wheel base if reduced_len $\%=1$.

$$
R L=T L-((T L-W B L) * \text { reduced_len } \%)
$$

Note that the company currently uses the PPM to solve a simplified version of the problem which does not consider the newly introduced LPM parameters. Given a set of machines returned by the PPM, truck drivers rely on their experience to generate a load plan and do not explicitly consider constraints associated with the introduced parameters. These parameters are thus specific to the LPM and have not previously been exploited by the company. Therefore, a careful calibration of these parameters is essential to 
generate a model well tuned to the problem-specific scenario and capable of efficiently classifying load plans.

In a scenario where LPM is called repeatedly, the load plan, whether it is feasible or not, can be stored in a memory structure referred to as TRIE [8]. TRIE avoids evaluating routes that have already had their load plan checked, significantly accelerating the runtime of the overall algorithm (vehicle routing plus LPM).

\section{COMPUTATIONAL STUDY}

This section presents computational experiments to validate the performance of the developed approach and compare the LPM with the current practice. The company provided us with the dimensions and characteristics of their machines: length, width, height, weight, wheelbase length and predicted percentage. A set of 196 candidate load plans, including both feasible and infeasible configurations, was also provided with the classification of experienced human operators.

Table 1 provides the necessary parameters for the LPM. The value of max_weight should be selected so as to remain compliant with national regulations. We set this value to 22 tons in our experiments which corresponds to the value stipulated in Belgian legislation. We should note here that the values of these parameters will vary from country to country and have a significant impact on possible combinations. Using a random subset of the available instances, all the remaining parameters were calibrated using irace [10].

Table 1: LPM parameters and their values

\begin{tabular}{lc}
\hline Parameter & Value \\
\hline exW & $25 \mathrm{~cm}$ \\
max $_{w}$ & $132 \mathrm{~cm}$ \\
min_gNeck & $744 \mathrm{~cm}$ \\
reduced_len $\%$ & 0.47 \\
max_weight & 22 tons \\
\hline
\end{tabular}

The goal of the LPM is to classify load plans as (in)feasible with more precision than the PPM, which simplifies the real dimensions of machines. The LPM is thus a decision method which divides machine combinations into two classes: feasible and infeasible load plans. We compare the accuracy of three different methods: (i) the predicted percentage method currently employed by the company, (ii) the LPM-Base method which corresponds to the best-fit heuristic proposed by Burke et al. [2] for solving 2D packing problems and (iii) the LPM method which we adapted from LPM-Base by integrating all of the extra parameters described in Section 4. For the purpose of this comparison, Table 2 provides the confusion matrix of each method where columns TP, FP, TN and FN correspond to each classification method's number of true positives, false positives, true negatives and false negatives, respectively. We provide the precise meaning of these categories in Table 3.

To assess the performance of each method, well-known metrics to compare binary classifiers are calculated. The F1-score measures the method's accuracy and is calculated as per Equation (2).

$$
F 1-\text { Score }=\frac{T P}{T P+0.5(F P+F N)}
$$

Since F1-score do not take into account the number of true negatives, a second metric, Matthews Correlation Coefficient (MCC), is also employed and is calculated as per Equation (3).

$$
M C C=\frac{(T P \times T N)-(F P \times F N)}{\sqrt{(T P+F P)(T P+F N)(T N+F P)(T N+F N)}}
$$

Table 2: Confusion matrix of the loading methods.

\begin{tabular}{l|cccccc}
\hline $\begin{array}{l}\text { Loading } \\
\text { method }\end{array}$ & TP & FP & TN & FN & F1-score & MCC \\
\hline PPM & 74 & 16 & 83 & 23 & 0.791 & 0.603 \\
LPM-Base & 82 & 19 & 80 & 15 & 0.828 & 0.654 \\
LPM & 87 & 10 & 89 & 10 & 0.897 & 0.796 \\
\hline
\end{tabular}

\begin{tabular}{|c|c|c|c|}
\hline $\begin{array}{l}\text { Confusion } \\
\text { parameter }\end{array}$ & Abbreviation & $\begin{array}{c}\text { Method's } \\
\text { decision }\end{array}$ & Actuality \\
\hline True positive & $\mathrm{TP}$ & Feasible & Feasible \\
\hline False positive & $\mathrm{FP}$ & Feasible & Infeasible \\
\hline True negative & $\mathrm{TN}$ & Infeasible & Infeasible \\
\hline False negative & FN & Infeasible & Feasible \\
\hline
\end{tabular}

Table 3: Confusion categories.

The PPM obtains the worst results, misclassifying up to $20 \%$ of the considered instances. Since operators often assign a higher predicted percentage to machines, this method results in many feasible routes having a total predicted percentage greater than $100 \%$. This leads to many false negatives.

LPM-base considers machines as 2D rectangles, but does not take into account constraints related to the gooseneck or safety concerns in regards to width. Consequently, this method also results in a high number of false positives. In other words, many combinations that LPM-base considers feasible are not feasible in reality. Moreover, when ignoring the possibility of machine overlapping many feasible solutions are misclassified as infeasible, which helps explain most of LPM-Base's false negatives.

Although LPM does not correctly classify all instances, it exhibits better accuracy across all metrics and classifies approximately $90 \%$ of the instances correctly. By employing this method, the company is able to significantly improve their routing plans. Moreover, LPM was able to discover several feasible load plans which were unknown to the company's operators prior to this study.

When we take a closer look at the misclassifications by LPM, we observe that these mostly occur due to special cases where operators push the limits of safety restrictions by permitting extra width or increasing maximum weight. For false negative cases, some machines can make better use of the gooseneck in reality because the LPM method is overly restrictive when calculating the amount of space they can occupy over the gooseneck. Similarly, when overlapping machines may occupy a larger area in reality than allowed by the LPM method. 


\section{CONCLUSION}

This paper introduced the feasibility verification in machine packing problem (FVMP): a real-world container loading problem where one must decide whether or not a given set of non-identical irregular machines can be loaded onto a non-convex truck loading surface without violating several practical restrictions. The set of machines include a variety of forklifts, arms and cages as well as lifts that can be folded. The practical constraints include maximum weight restrictions, fixed orientation for machines and a minimum amount of empty space between the machines. Specific characteristics of the FVMP allow a partial overlapping of some machines which increases the possibility of feasibly loading the machines.

The FVMP must be solved very frequently within a computationally demanding routing algorithm. Therefore, the method for solving the FVMP should not only be highly accurate but also very efficient. In order to solve the FVMP, we developed an algorithm based on the best-fit placement heuristic which was originally introduced for solving the two-dimensional rectangular packing problem. The results of computational experiments conducted using real-world data indicated that the new algorithm outperforms the approach currently employed by the company.

Regarding future work, some of the defined parameters can be applied with different values depending on the machine type. For instance, for a forklift, everything which is not wheelbase (the fork) may be completely overlapped. By contrast, the articulated arm and cage of certain machines can only be partially overlapped, which means these machines needs to have a different percentage of overlapping.

The analysis and methods in this paper focused only on the feasibility verification of loading a set of machines associated with a given route onto a truck. Future research should also focus on problem extensions which combine loading and routing decisions and address them with integrated methods. This will necessarily result in the loading problem becoming even more complex since when routing trucks internationally each country may have different loading regulations which need to be adhered to.

\section{ACKNOWLEDGMENTS}

This research was supported by Internal Funds KU Leuven and by the Research Foundation Flanders (FWO) Strategic Basic Research project Data-driven logistics (S007318N). Editorial consultation provided by Luke Connolly (KU Leuven).

\section{REFERENCES}

[1] Andreas Bortfeldt and Gerhard Wäscher. 2013. Constraints in container loadingA state-of-the-art review. European fournal of Operational Research 229, 1 (2013), $1-20$.

[2] Edmund K Burke, Graham Kendall, and Glenn Whitwell. 2004. A new placement heuristic for the orthogonal stock-cutting problem. Operations Research 52, 4 (2004), 655-671.

[3] Oliviana Xavier do Nascimento, Thiago Alves de Queiroz, and Leonardo Junqueira. 2021. Practical constraints in the container loading problem: Comprehensive formulations and exact algorithm. Computers \& Operations Research 128 (2021), 105186.

[4] Giorgio Fasano. 2014. Tetris-like items. In Solving Non-standard Packing Problems by Global Optimization and Heuristics. Springer, 7-26.

[5] Shinji Imahori and Mutsunori Yagiura. 2010. The best-fit heuristic for the rectangular strip packing problem: An efficient implementation and the worst-case approximation ratio. Computers \& Operations Research 37, 2 (2010), 325-333.

[6] Rodolfo Ranck Júnior, Horacio Hideki Yanasse, Reinaldo Morabito, and Leonardo Junqueira. 2019. A hybrid approach for a multi-compartment container loading problem. Expert Systems with Applications 137 (2019), 471-492.

[7] Aline AS Leao, Franklina MB Toledo, José Fernando Oliveira, Maria Antónia Carravilla, and Ramón Alvarez-Valdés. 2020. Irregular packing problems: a review of mathematical models. European fournal of Operational Research 282, 3 (2020), 803-822.

[8] Stephen CH Leung, Jiemin Zheng, Defu Zhang, and Xiyue Zhou. 2010. Simulated annealing for the vehicle routing problem with two-dimensional loading constraints. Flexible Services and Manufacturing fournal 22, 1-2 (2010), 61-82.

[9] I Litvinchev, A Pankratov, and T Romanova. 2019. 3D irregular packing in an optimized cuboid container. IFAC-PapersOnLine 52, 13 (2019), 2014-2019.

[10] Manuel López-Ibáñez, Jérémie Dubois-Lacoste, Leslie Pérez Cáceres, Mauro Birattari, and Thomas Stützle. 2016. The irace package: Iterated racing for automatic algorithm configuration. Operations Research Perspectives 3 (2016), 43-58.

[11] Hanne Pollaris, Kris Braekers, An Caris, Gerrit K Janssens, and Sabine Limbourg. 2015. Vehicle routing problems with loading constraints: state-of-the-art and future directions. OR Spectrum 37, 2 (2015), 297-330.

[12] Balasubramanian Ram. 1992. The pallet loading problem: A survey. International fournal of Production Economics 28, 2 (1992), 217-225.

[13] Thibaut Vidal, Teodor Gabriel Crainic, Michel Gendreau, Nadia Lahrichi, and Walter Rei. 2012. A hybrid genetic algorithm for multidepot and periodic vehicle routing problems. Operations Research 60, 3 (2012), 611-624.

[14] Gerhard Wäscher, Heike Haußner, and Holger Schumann. 2007. An improved typology of cutting and packing problems. European journal of operational research 183, 3 (2007), 1109-1130.

[15] Xiaozhou Zhao, Julia A Bennell, Tolga Bektaş, and Kath Dowsland. 2016. A comparative review of 3D container loading algorithms. International Transactions in Operational Research 23, 1-2 (2016), 287-320. 